\title{
ON THE SUBPARTITIONS OF THE ORDINARY PARTITIONS
}

\author{
BYUNGCHAN KIM \\ Dedicated to George E. Andrews for his seventieth birthday
}

\begin{abstract}
Let $a_{1} \geq a_{2} \geq \cdots \geq a_{\ell}$ be an ordinary partition. A subpartition with gap $d$ of an ordinary partition is defined as the longest sequence satisfying $a_{1}>a_{2}>$ $\cdots>a_{s}$ and $a_{s}>a_{s+1}$, where $a_{i}-a_{j} \geq d$ for all $i<j \leq s$. This is a generalization of the Rogers-Ramanujan subpartition which was introduced by L. Kolitsch. In this note, we will study various properties of the subpartition and as an application, we will give a combinatorial proof of two entries which are in Ramanujan's lost notebook.
\end{abstract}

\section{INTRODUCTION}

Let $a_{1} \geq a_{2} \geq \cdots \geq a_{\ell}$ be an ordinary partition. In a recent paper [7], L. Kolitsch introduced the Rogers-Ramanujan subpartitions and established their connection to other partitions. The Rogers-Ramanujan subpartition is the longest sequence satisfying $a_{1}>a_{2}>\cdots>a_{s}$ and $a_{s}>a_{s+1}$, where $a_{i}-a_{j} \geq 2$ for all $i<j \leq s$. In this note, we will generalize his result with an arbitrary gap condition and will study their connections to other partitions. Let us fix a positive integer $d$. Then, for a given partition, a subpartition with gap $d$ is defined as the longest sequence satisfying $a_{1}>$ $a_{2}>\cdots>a_{s}$ and $a_{s}>a_{s+1}$, where $a_{i}-a_{j} \geq d$ for all $i<j \leq s$. Note that Kolitsch's Rogers-Ramanujan subpartition is the case $d=2$. For convenience, we will define the subpartition of the empty partition as the empty partition. We define the length of the subpartition with gap $d$ as the number of parts in the subpartition. When the gap $d$ is clear in the context, we will say the subpartition instead of the subpartition with gap $d$. In Section 2, we will give a generating function of the ordinary partition that keeps track of the length of the subpartition with gap $d$. In Section 3, we will study

Key words and phrases. Partition, subpartition, partial theta function.

Mathematics Subject Classification (2000): 11P81, 05A17. 
their connection to the partial theta function, which is of the form

$$
\sum_{n=0}^{\infty}(-1)^{n} q^{n(n-1) / 2} x^{n}
$$

by attaching a proper weight to the generating function. In Section 4, we will focus on the subpartition with gap 1. By using the results from Section 2 and 3, we will give combinatorial proofs of the identities:

$$
\begin{aligned}
\frac{1}{(q)_{\infty}^{2}} \sum_{n=0}^{\infty}(-1)^{n} q^{\left(n^{2}+n\right) / 2} & =\sum_{n=0}^{\infty} \frac{q^{n}}{(q)_{n}^{2}} \\
\frac{1}{(q)_{\infty}^{2}}\left(1+2 \sum_{n=1}^{\infty}(-1)^{n} q^{\left(n^{2}+n\right) / 2}\right) & =\sum_{n=0}^{\infty} \frac{q^{2 n}}{(q)_{n}^{2}},
\end{aligned}
$$

which are entries in Ramanujan's lost notebook [8] [3, p. 19, Entry 1.4 .10 and Entry 1.4.11]. Here and in the sequel, we use the customary notation for $q$-series:

$$
\begin{aligned}
& (a)_{0}:=(a ; q)_{0}:=1 \\
& (a)_{n}:=(a ; q)_{n}:=(1-a)(1-a q) \cdots\left(1-a q^{n-1}\right), n \geq 1
\end{aligned}
$$

and

$$
(a)_{\infty}:=(a ; q)_{\infty}:=\lim _{n \rightarrow \infty}(a ; q)_{n},|q|<1 .
$$

We will conclude this note with some comments on possible future projects.

\section{A Generating FunCtion}

For a given partition $\lambda$, we always write it in the form $a_{1} \geq a_{2} \geq \cdots \geq a_{\ell}$. Before finding a generating function, we need to define some notation. Let us fix a positive integer $d$ and define, for each nonnegative integer $k$,

$$
S_{d, k}=\left\{\begin{array}{l}
1+(1+d)+(1+2 d)+\cdots(k-1) d+1=\frac{d k^{2}-(d-2) k}{2}, \text { if } k \neq 0, \\
0, \text { if } k=0
\end{array}\right.
$$

Then for a given partition $\lambda$, there are three cases:

(I) There is no subpartition in $\lambda$. 
(II) The subpartition of $\lambda$ is $\lambda$. In this case, we will say the partition $\lambda$ is a complete partition after Kolitsch.

(III) $\lambda$ is not complete and it has a subpartition with length $\ell$.

For the case (I), i.e. to have no subpartition in $\lambda$, we should have $a_{1}=a_{2}$. By using a standard argument [1, chap. 1], we can easily see that

$$
\sum_{i=1}^{\infty} \frac{q^{2 i}}{(q)_{i}}
$$

generates such partitions.

For the case (II), i.e. $\lambda$ is a complete partition, the gaps between successive parts of $\lambda$ should be at least $d$. Such partitions are generated by

$$
\sum_{\ell=0}^{\infty} \frac{q^{S_{d, \ell}}}{(q)_{\ell}} .
$$

Note that the length of the subpartition in the above is $\ell$.

For the case (III), suppose that a given partition $\lambda$ has the subpartition with length $\ell$ and $a_{\ell}=j$. Note that since $\lambda$ is not a complete partition, there are at least $\ell+1$ parts in $\lambda$ and, by definition, $a_{\ell}>a_{\ell+1}$. Then, there are two possibilities:

(i) $a_{\ell}-a_{\ell+1}$ is less than $d$.

(ii) $a_{\ell}-a_{\ell+1} \geq d$, but $a_{\ell+1}=a_{\ell+2}$. 
For the case (i), we have the generating function

$$
\begin{aligned}
& \sum_{\ell=1}^{\infty} \frac{q^{S_{d, \ell}}}{(q)_{\ell-1}}\left(\sum_{j=2}^{\infty} \frac{q^{(\ell+1)(j-1)}}{(q)_{j-1}}+\cdots+\sum_{j=d}^{\infty} \frac{q^{\ell(j-1)+(j-d+1)}}{(q)_{j-d+1}}\right) \\
& =\sum_{\ell=1}^{\infty} \frac{q^{S_{d, \ell}}}{(q)_{\ell-1}}\left(\sum_{n=1}^{\infty} \frac{q^{(\ell+1) n}}{(q)_{n}}+\cdots+q^{\ell(d-2)} \sum_{n=1}^{\infty} \frac{q^{(\ell+1) n}}{(q)_{n}}\right) \\
& =\sum_{\ell=1}^{\infty} \frac{q^{S_{d, \ell}}\left(1-q^{\ell(d-1)}\right)}{(q)_{\ell}} \sum_{n=1}^{\infty} \frac{q^{(\ell+1) n}}{(q)_{n}} \\
& =\sum_{\ell=1}^{\infty} \frac{q^{S_{d, \ell}}\left(1-q^{\ell(d-1)}\right)}{(q)_{\ell}}\left(\frac{1}{\left(q^{\ell+1}\right)_{\infty}}-1\right) \\
& =\sum_{\ell=1}^{\infty} \frac{q^{S_{d, \ell}}\left(1-q^{\ell(d-1)}\right)}{(q)_{\infty}}-\sum_{\ell=1}^{\infty} \frac{q^{S_{d, \ell}}\left(1-q^{\ell(d-1)}\right)}{(q)_{\ell}},
\end{aligned}
$$

where in the penultimate line we used the $q$-binomial theorem $[5$, p. 8]. For the case (ii), we have the generating function

$$
\begin{aligned}
& \sum_{\ell=1}^{\infty} \frac{q^{S_{d, \ell}}}{(q)_{\ell-1}} \sum_{j=d+1}^{\infty} q^{\ell(j-1)} \sum_{i=1}^{j-d} \frac{q^{2 i}}{(q)_{i}} \\
& =\sum_{\ell=1}^{\infty} \frac{q^{S_{d, \ell}}}{(q)_{\ell-1}} \sum_{i=1}^{\infty} \sum_{j=d+i}^{\infty} q^{\ell(j-1)} \frac{q^{2 i}}{(q)_{i}} \\
& =\sum_{\ell=1}^{\infty} \frac{q^{S_{d, \ell}+\ell(d-1)}}{(q)_{\ell}} \sum_{i=1}^{\infty} \frac{q^{(\ell+2) i}}{(q)_{i}} \\
& =\sum_{\ell=1}^{\infty} \frac{q^{S_{d, \ell}+\ell(d-1)}}{(q)_{\ell}}\left(\frac{1}{\left(q^{\ell+2}\right)_{\infty}}-1\right),
\end{aligned}
$$

by the $q$-binomial theorem. 
Since all partitions fall into one of the above three cases, the sum of the above generating functions $((3),(4),(5)$, and $(6))$ should be $\frac{1}{(q)_{\infty}}$. Thus, we have

$$
\begin{aligned}
\frac{1}{(q)_{\infty}} & =\sum_{i=1}^{\infty} \frac{q^{2 i}}{(q)_{i}}+\sum_{\ell=0}^{\infty} \frac{q^{S_{d, \ell}}}{(q)_{\ell}} \\
& +\sum_{\ell=1}^{\infty} \frac{q^{S_{d, \ell}}\left(1-q^{\ell(d-1)}\right)}{(q)_{\infty}}-\sum_{\ell=1}^{\infty} \frac{q^{S_{d, \ell}}\left(1-q^{\ell(d-1)}\right)}{(q)_{\ell}}+\sum_{\ell=1}^{\infty} \frac{q^{S_{d, \ell}+\ell(d-1)}}{(q)_{\ell}}\left(\frac{1}{\left(q^{\ell+2}\right)_{\infty}}-1\right) \\
& =\sum_{\ell=0}^{\infty} \frac{q^{S_{d, \ell}+\ell(d-1)}}{(q)_{\ell}\left(q^{\ell+2}\right)_{\infty}}+\sum_{\ell=1}^{\infty} \frac{q^{S_{d, \ell}}\left(1-q^{\ell(d-1)}\right)}{(q)_{\infty}}
\end{aligned}
$$

since

$$
\sum_{i=1}^{\infty} \frac{q^{2 i}}{(q)_{i}}=\frac{1}{\left(q^{2}\right)_{\infty}}-1
$$

by the $q$-binomial theorem. Thus, we have proved our first theorem.

Theorem 1. Let $\ell$ be the length of the subpartition with gap $d$, then we have

$$
\frac{1}{(q)_{\infty}}=\sum_{\ell=0}^{\infty} \frac{q^{S_{d, \ell}+\ell(d-1)}}{(q)_{\ell}\left(q^{\ell+2}\right)_{\infty}}+\sum_{\ell=1}^{\infty} \frac{q^{S_{d, \ell}}\left(1-q^{\ell(d-1)}\right)}{(q)_{\infty}} .
$$

Remark. An analytic proof of Theorem 1 is very simple; thus we will omit it. Note that, by setting $d=2$, we can recover Kolitsch's Theorem 1 .

Define $p(n, \ell, d)$ to be the number of partitions of $n$ having a subpartition of length $\ell$ with gap $d$. Then, by comparing coefficients of $q^{n}$ in the (7), we can easily deduce that

Corollary 2. For all nonnegative integers $n$ and $\ell$ and a positive integer $d$, we have

$$
p(n, \ell, d)=\left\{\begin{array}{l}
p\left(n-S_{d, \ell}\right)-p\left(n-\left(S_{d, \ell}+\ell(d-1)\right)\right) \text { if } d>1, \\
p\left(n-\frac{\ell(\ell+1)}{2}\right)-p\left(n-\frac{(\ell+1)(\ell+2)}{2}\right) \text { if } d=1 .
\end{array}\right.
$$

\section{Subpartitions With PARITy CONDITION}

Let us define $p_{e}(n, d)$ to be the sum $\sum_{\ell \text { even }} p(n, \ell, d)$, i.e. the number of partitions of $n$ that have subpartitions with even lengths, and similarly for $p_{o}(n, d)$. Then, we 
have

$$
\begin{aligned}
\sum_{n=0}^{\infty}\left(p_{e}(n, d)-p_{o}(n, d)\right) q^{n} & =\sum_{\ell=0}^{\infty} \frac{(-1)^{\ell} q^{S_{d, \ell}+\ell(d-1)}}{(q)_{\ell}\left(q^{\ell+2}\right)_{\infty}}+\sum_{\ell=1}^{\infty} \frac{(-1)^{\ell} q^{S_{d, \ell}}\left(1-q^{\ell(d-1)}\right)}{(q)_{\infty}} \\
& =\frac{1}{(q)_{\infty}}\left(\sum_{\ell=1}^{\infty}(-1)^{\ell} q^{S_{d, \ell}}\left(1-q^{\ell(d-1)}\right)+\sum_{\ell=0}^{\infty}(-1)^{\ell} q^{S_{d, \ell}+\ell(d-1)}\left(1-q^{\ell+1}\right)\right) \\
& =\frac{1}{(q ; q)_{\infty}}\left(1+2 \sum_{\ell=1}^{\infty}(-1)^{\ell} q^{S_{d, \ell}}\right) \\
& =\frac{1}{(q)_{\infty}}\left(2 \sum_{\ell=0}^{\infty}(-1)^{\ell} q^{S_{d, \ell}}-1\right)
\end{aligned}
$$

Note that when $d=2,1+2 \sum_{k=1}^{\infty}(-1)^{k} q^{S_{d, k}}$ becomes a theta function that implies Kolitsch's Theorem 4. For other d's, $\sum_{k=0}^{\infty}(-1)^{k} q^{S_{d, k}}$ is a partial theta function of the form,

$$
\sum_{k=0}^{\infty}(-1)^{k} q^{\frac{d k^{2}-(d-2) k}{2}}
$$

Since, $p_{e}(n, d)+p_{o}(n, d)=p(n)$, we have

$$
\sum_{n=0}^{\infty} p_{e}(n, d) q^{n}=\frac{1}{(q)_{\infty}} \sum_{k=0}^{\infty}(-1)^{k} q^{S_{d, k}} .
$$

By replacing $a$ and $q$ by $q$ and $q^{d}$, respectively, in the identity [4, eqn (2.1b)],

$$
\sum_{k=0}^{\infty}(-1)^{k} a^{k} q^{\left(k^{2}-k\right) / 2}=(a)_{\infty} \sum_{n=0}^{\infty} \frac{a^{n} q^{n^{2}}}{(q)_{n}(a)_{n}},
$$

we obtain

$$
\sum_{n=0}^{\infty} p_{e}(n, d) q^{n}=\frac{\left(q ; q^{d}\right)_{\infty}}{(q)_{\infty}} \sum_{m=0}^{\infty} \frac{q^{d m^{2}+m}}{\left(q^{d} ; q^{d}\right)_{m}\left(q ; q^{d}\right)_{m}}
$$

We have the following partition theoretic interpretation of (10).

Theorem 3. In the case of $d \geq 2$, the number of partitions of $n$ with an even length subpartition with gap $d$ is the same as the number of partitions of $n$ such that the parts which are congruent to 1 modulo d have the following property: Consider the d-modular diagram of the partition, which consists of such parts. If it has the Durfee square of a side $k$, then the largest part of the partition below the Durfee square should be less than or equal to $d(k-1)+1$. In the case of $d=1$, the number of partitions of $n$ with 
subpartitions of even length is the same as the number of partitions of $n$ that have the following property: If it has the Durfee square of a side $k$, then the number of parts in the partition on the right side of the Durfee square is $k$.

\section{Subpartitions With Gap 1}

In this section, we will investigate the subpartitions with gap 1. By (8) and (9) in Section 3, we have

$$
\begin{aligned}
\sum_{n=0}^{\infty}\left(p_{e}(n, 1)-p_{o}(n, 1)\right) q^{n} & =\frac{1}{(q)_{\infty}}\left(1+2 \sum_{k=1}^{\infty}(-1)^{k} q^{\left(k^{2}+k\right) / 2}\right) \\
\sum_{n=0}^{\infty} p_{e}(n, 1) q^{n} & =\frac{1}{(q)_{\infty}} \sum_{k=0}^{\infty}(-1)^{k} q^{\left(k^{2}+k\right) / 2}
\end{aligned}
$$

Thus, Entry 1.4.10 and 1.4.11 of [3, p. 19] are equivalent to the following identities:

$$
\begin{aligned}
\sum_{n \geq 0} p_{e}(n, 1) q^{n} & =\sum_{n=0}^{\infty} \frac{q^{n}}{(q)_{n}}\left(q^{n+1}\right)_{\infty}, \\
\sum_{n \geq 0}\left(p_{e}(n, 1)-p_{o}(n, 1)\right) q^{n} & =\sum_{n=0}^{\infty} \frac{q^{2 n}}{(q)_{n}}\left(q^{n+1}\right)_{\infty} .
\end{aligned}
$$

Now we will give a combinatorial proof for the above identities. Throughout the proofs, $t(\lambda)$ denotes the number of parts in $\lambda$.

Proof of (11). Note that

$$
\frac{q^{n}}{(q)_{n}}\left(q^{n+1}\right)_{\infty}
$$

generates partition pairs $(\pi(n), \sigma(n))$, where $\pi(n)$ is a partition with $\left(n=\pi_{1}(n), \pi_{2}(n)\right.$, $\left.\ldots, \pi_{t}(n)\right)$, and $\sigma(n)$ is a partition into distinct parts such that the smallest part is larger than $n$, and the exponent of $(-1)$ is $t(\sigma(n))$. For a given partition $\lambda$, suppose that $\lambda$ has the subpartition of length $\ell$. Then, $\lambda$ is of the form $\lambda_{1}>\lambda_{2}>\cdots>\lambda_{\ell}>$ $\lambda_{\ell+1} \geq \lambda_{\ell+2} \geq \cdots$. Thus, in the right hand side of (11), $\lambda$ is generated $\ell+1$ times as $\left(\pi\left(\lambda_{1}\right), \emptyset\right),\left(\pi\left(\lambda_{2}\right), \sigma\left(\lambda_{2}\right)\right), \ldots$, and $\left(\pi\left(\lambda_{\ell+1}\right), \sigma\left(\lambda_{\ell+1}\right)\right)$. Note that, in fact, $\lambda_{\ell+1}=\lambda_{\ell+2}$. If not, the length of the subpartition should be bigger than $\ell$. Thus, $\lambda$ is not of the form $\left(\pi\left(\lambda_{\ell+2}\right), \sigma\left(\lambda_{\ell+2}\right)\right)$. Note also that the exponent of $(-1)$ in the previous partition pairs is $(-1)^{0},(-1)^{1}, \ldots,(-1)^{\ell}$, respectively. Thus, their sum is 1 if $\ell$ is even and is 
0 if $\ell$ is odd. Thus, in the right side of (11), after cancellation, we are left with the partitions that have subpartitions with even length.

Proof of (12). Note that

$$
\frac{q^{2 n}}{(q)_{n}}\left(q^{n+1}\right)_{\infty}
$$

generates partition pairs $(\pi(n), \sigma(n))$, where $\pi(n)$ is a partition with $\pi_{1}(n)=\pi_{2}(n)=n$, $\sigma(n)$ is a partition into distinct parts such that the smallest part is larger than $n$, and the exponent of $(-1)$ is $t(\sigma(n))$. For a given partition $\lambda$, suppose that $\lambda$ has the subpartition with length $\ell$. Then, as before, $\lambda$ is of the form $\lambda_{1}>\lambda_{2}>\cdots>\lambda_{\ell}>\lambda_{\ell+1} \geq \lambda_{\ell+2} \geq \cdots$. Recall that $\lambda_{\ell+1}=\lambda_{\ell+2}$. Thus, in the right side, $\lambda$ is generated as $\left(\pi\left(\lambda_{\ell+1}\right), \sigma\left(\lambda_{\ell+1}\right)\right)$. Since the exponent of $(-1)$ is $\ell$ in this partition pair, we are done.

Note that the right side of (1) is a generating function of the number of stacks with summit. For the definition of the stack of summit and its proof, consult the paper of Andrews [2]. For other combinatorial proofs of Entry 1.4.10 and Entry 1.4.11, examine the work of A.J. Yee [9] or the previous work of the author [6].

Next, we will obtain another generating function for $p_{e}(n, 1)-p_{o}(n, 1)$ by using a simple Durfee square argument. For a given partition $\lambda$, let $\lambda^{r}$ be the conjugate of the partition in the right side of the Durfee square and $\lambda^{b}$ be the partition below the Durfee square. Let $s(\lambda)$ be the side of the Durfee square of $\lambda$. Then, the coefficient of $q^{n}$ of

$$
2 \sum_{k=0}^{\infty} \frac{q^{k^{2}+k}}{(q)_{k}^{2}}-\frac{1}{(q)_{\infty}}=2 \sum_{k=0}^{\infty} \frac{q^{k^{2}+k}}{(q)_{k}^{2}}-\sum_{k=0}^{\infty} \frac{q^{k^{2}}}{(q)_{k}^{2}}
$$

is (the number of partitions of $n$ such that $\lambda_{1}^{b}=s(\lambda)$ ) minus (the number of partitions of $n$ such that $\left.\lambda_{1}^{r}=s(\lambda)\right)$ minus $p(n)$, by symmetry. Since $\lambda$ with $\lambda_{1}^{b}<s(\lambda)$ is not generated by $\sum_{k=0}^{\infty} \frac{q^{k^{2}+k}}{(q)_{k}^{2}}$ and we count $\lambda$ twice if $\lambda_{1}^{r}=\lambda_{1}^{b}=s(\lambda)$, we have

$$
2 \sum_{k=0}^{\infty} \frac{q^{k^{2}+k}}{(q)_{k}^{2}}-\sum_{k=0}^{\infty} \frac{q^{k^{2}}}{(q)_{k}^{2}}=1+\sum_{k=1}^{\infty} \frac{q^{k^{2}+2 k}}{(q)_{k}^{2}}-\sum_{k=1}^{\infty} \frac{q^{k^{2}}}{(q)_{k-1}^{2}}
$$


In summary, we have

$$
\begin{aligned}
\sum_{n \geq 0}\left(p_{e}(n, 1)-p_{o}(n, 1)\right) q^{n} & =\frac{1}{(q)_{\infty}}\left(1+2 \sum_{k=1}^{\infty}(-1)^{k} q^{\left(k^{2}+k\right) / 2}\right) \\
& =1+\sum_{k=1}^{\infty} \frac{q^{k^{2}+2 k}}{(q)_{k}^{2}}-\sum_{k=1}^{\infty} \frac{q^{k^{2}}}{(q)_{k-1}^{2}} \\
& =(1-q) \sum_{k=0}^{\infty} \frac{q^{k^{2}+2 k}}{(q ; q)_{k}^{2}}
\end{aligned}
$$

Therefore, we have proved the following theorem.

Theorem 4. The difference between the number of partitions of $n$ with subpartition (with gap 1) of even length and those of odd length is the number of partitions $\lambda$ of $n$ satisfying $\lambda_{1}^{r}=\lambda_{1}^{b}=s(\lambda)$ and 1 is not a part of $\lambda^{b}$.

As an immediate corollary, we have

$$
p_{e}(n, 1) \geq p_{o}(n, 1), \text { for all } n \geq 2 \text {. }
$$

Note that equality holds if and only if $n=2$.

Next, we will investigate the parity of $p_{e}(n, 1)$. Applying Jacobi's identity and the pentagonal number theorem $[5$, p. 14,12$]$, we find that

$$
\begin{aligned}
\sum_{n \geq 0} p_{e}(n, 1) q^{n} & \equiv \frac{1}{(q)_{\infty}} \sum_{n \geq 0} q^{\left(n^{2}+n\right) / 2} \quad(\bmod 2) \\
& \equiv \frac{(q)_{\infty}^{3}}{(q)_{\infty}}(\bmod 2) \\
& \equiv\left(q^{2} ; q^{2}\right)_{\infty} \quad(\bmod 2) \\
& \equiv \sum_{n=-\infty}^{\infty} q^{n(3 n-1)}(\bmod 2) .
\end{aligned}
$$

Thus, we can conclude that $p_{e}(n, 1)$ is almost always even. Hence, we have proved the following theorem.

Theorem 5. For all nonnegative integer n, we have

$p_{e}(n, 1) \equiv \begin{cases}1 & (\bmod 2), \text { if } n \text { is of the form } n(3 n \pm 1) \text { for some nonnegative integer } n \\ 0 & (\bmod 2), \text { otherwise. }\end{cases}$ 


\section{Concluding Remarks}

By Corollary 2, we have

$$
p(n, \ell, 1)=p\left(n-\frac{\left(\ell^{2}+\ell\right)}{2}\right)-p\left(n-\frac{(\ell+1)(\ell+2)}{2}\right) .
$$

From the famous Ramanujan congruences, we can deduce the following trivial congruences for $p(n, \ell, 1)$ :

$$
\begin{aligned}
& p(5 n+4,5 k+4,1) \equiv 0 \quad(\bmod 5), \\
& p(7 n+5,7 k+6,1) \equiv 0 \quad(\bmod 7), \\
& p(11 n+6,11 k+10,1) \equiv 0 \quad(\bmod 11),
\end{aligned}
$$

for all nonnegative integer $n$ and $k$. It would be very interesting to find a congruence for $p(n, \ell, d)$ besides the congruences that are inherited by the congruences of the ordinary partition function.

Secondly, are there interesting properties of the subpartitions of other partitions? For example, let define the subpartition of partitions into distinct parts as the longest sequence with the gap between the parts being exactly 1 . Then, by a similar argument that we used to prove Theorem 1, we can easily prove that

$$
(-q)_{\infty}=\sum_{\ell=0}^{\infty} q^{\left(\ell^{2}+\ell\right) / 2}+\sum_{\ell=1}^{\infty} q^{\left(\ell^{2}+\ell\right) / 2} \sum_{i=1}^{\infty} q^{i \ell}(-q)_{i-1}
$$

and

$$
(-q)_{\infty}=\sum_{\ell=0}^{\infty} \frac{q^{\left(\ell^{2}+\ell\right) / 2}}{1-q^{\ell}}+\sum_{\ell=1}^{\infty} q^{\left(\ell^{2}+\ell\right) / 2} \sum_{i=1}^{\infty} q^{i \ell}\left((-q)_{i-1}-1\right)
$$

where $\ell$ is the length of the subpartition. It appears that (15) and (16) do not appear in the literature of $q$-series. Can we prove analytically (15) or (16)?

\section{ACKNOWLEDGMENTS}

The author would like to thank Bruce Berndt for his encouragement and help. 


\section{REFERENCES}

[1] G.E. Andrews, The theory of partitions, Addison-Wesley, Reading, MA, 1976; reissued by Cambridge University Press, Cambridge, 1984.

[2] G.E. Andrews, Ramanujan's "lost" notebook. IV. stacks and alternating parity in partitions, Adv. in Math., 53 (1984), 55- 74.

[3] G.E. Andrews and B.C. Berndt, Ramanujan's lost notebook, part II, Springer, To appear.

[4] G.E. Andrews and S.O. Warnaar, The product of partial theta functions, Adv. in App. Math., 39 (2007), 116-120.

[5] B.C. Berndt, Number theory in the spirit of Ramanujan, American Mathematical Society, Providence, RI, 2006.

[6] B. Kim, Combinatorial proofs of certain identities involving partial theta functions, Internat. J. Number Thy, to appear.

[7] L.W. Kolitsch, Rogers-Ramanujan subpartitions and their connections to other partition, Ramanujan J., 2008, 163-167.

[8] S. Ramanujan, The lost notebook and other unpublished papers, Narosa, New Delhi, 1988.

[9] A.J. Yee, Bijective proofs of a theorem of Fine and related partition identities, Internat. J. Number Thy, to appear.

Department of Mathematics, University of Illinois, 1409 West green street, URBANA, IL 61801, USA

E-mail address: bkim4@illinois.edu 